\title{
Le routier de Piarres Detcheverry, 1677. Un aperçu de la présence basque dans la baie des Chaleurs au XVII ${ }^{\mathrm{e}}$ siècle
}

\section{Brad Loewen et Miren Egaña Goya}

Volume 68, numéro 1-2, été-automne 2014

URI : https://id.erudit.org/iderudit/1032022ar

DOI : https://doi.org/10.7202/1032022ar

Aller au sommaire du numéro

Éditeur(s)

Institut d'histoire de l’Amérique française

ISSN

0035-2357 (imprimé)

1492-1383 (numérique)

Découvrir la revue

Citer cet article

Loewen, B. \& Egaña Goya, M. (2014). Le routier de Piarres Detcheverry, 1677. Un aperçu de la présence basque dans la baie des Chaleurs au XVII ${ }^{\mathrm{e}}$ siècle. Revue d'histoire de l'Amérique française, 68(1-2), 125-151.

https://doi.org/10.7202/1032022ar
Résumé de l'article

Le routier en langue basque de Piarres Detcheverry, publié à Bayonne en 1677, contient des descriptions de la pointe de la péninsule gaspésienne et surtout la baie des Chaleurs. L'examen de ces passages, accompagnés de leur traduction, révèle un portrait inédit de la présence basque dans ces régions. La conjoncture des pêches basques en Nouvelle-France dans la seconde moitié du $\mathrm{XVII}^{\mathrm{e}}$ siècle forme le contexte historique du routier. L'ouvrage contient aussi des indices de rapports entre Basques et Autochtones, à partir d'une toponymie majoritairement micmaque pour les lieux d'établissement basque. On peut enfin se demander pourquoi les écrits français n'ont pas mis plus en lumière cette présence basque. Nous proposons que la nationalité espagnole de plusieurs de ces pêcheurs a contribué à leur faible visibilité dans les archives coloniales, dans un contexte historique où décrire l'Autre légitimait sa présence.
Tous droits réservés @ Institut d'histoire de l’Amérique française, 2015

Ce document est protégé par la loi sur le droit d'auteur. L'utilisation des services d'Érudit (y compris la reproduction) est assujettie à sa politique d'utilisation que vous pouvez consulter en ligne.

https://apropos.erudit.org/fr/usagers/politique-dutilisation/ 


\title{
Le routier de Piarres Detcheverry, I677. Un aperçu de la présence basque dans la baie des Chaleurs au XVII ${ }^{\mathrm{e}}$ siècle
}

\author{
BRAD LOEWEN \\ Université de Montréal \\ Miren Egaña Goya \\ Sociedad de Ciencias Aranzadi, Donostia-San Sebastián
}

\begin{abstract}
RÉSUMÉ • Le routier en langue basque de Piarres Detcheverry, publié à Bayonne en 1677, contient des descriptions de la pointe de la péninsule gaspésienne et surtout la baie des Chaleurs. L'examen de ces passages, accompagnés de leur traduction, révèle un portrait inédit de la présence basque dans ces régions. La conjoncture des pêches basques en Nouvelle-France dans la seconde moitié du XVIle siècle forme le contexte historique du routier. L'ouvrage contient aussi des indices de rapports entre Basques et Autochtones, à partir d'une toponymie majoritairement micmaque pour les lieux d'établissement basque. On peut enfin se demander pourquoi les écrits français n'ont pas mis plus en lumière cette présence basque. Nous proposons que la nationalité espagnole de plusieurs de ces pêcheurs a contribué à leur faible visibilité dans les archives coloniales, dans un contexte historique où décrire l'Autre légitimait sa présence.
\end{abstract}

ABSTRACT - Piarres Detcheverry's pilot book, written in Basque and published in Bayonne in 1677, contains descriptions of the point of the Gaspé Peninsula and especially Chaleur Bay. Examination of these passages, accompanied by their French translation, shows an original portrait of the Basque presence in these regions. The specific conjuncture of the Basque fisheries in New France during the second half of the $17^{\text {th }}$ century forms the pilot book's historical context. The work also contains clues to the relation between the Basque and Native communities, based on a primarily Mi'kmaq toponymy of the locations where the Basques were established. We may ask why French sources have not shed more light on this Basque presence and we suggest that, in a context where describing the Other legitimised 
their presence, the Spanish nationality of many of these fishing crews underlies their faint visibility in colonial archives.

L a présence de pêcheurs basques dans la baie des Chaleurs a été régulièrement relevée aux XVII ${ }^{\mathrm{e}}$ et $\mathrm{XVIII}^{\mathrm{e}}$ siècles ${ }^{1}$. Ainsi, Samuel de Champlain mentionne les Basques à maintes reprises, notamment en précisant que jusqu'à onze de leurs navires s'installaient chaque été à Miscou dans les années $1620^{2}$. Un siècle plus tard, de 1719 à 1730 , il est connu qu'un capitaine du Labourd pêchait à Pabos et, qu'au cours des années 1730 et 1740, une trentaine de navires marchands basques y firent escale, profitant d'une route maritime tracée depuis longtemps entre le Pays Basque et la baie des Chaleurs ${ }^{3}$. C'est d'ailleurs auprès des maîtres d'œuvre basques que des entrepreneurs canadiens trouvèrent un savoirfaire pour l'apprêt du poisson, la morue salée et séchée ${ }^{4}$. Loin d'être des visiteurs de passage, les Basques ont aussi laissé un patrimoine durable chez les Autochtones de la région, qui utilisaient un pidgin de traite «à moitié basque ${ }^{5}$ ».

À partir des différents écrits, un portrait des activités et des occupations des Basques dans la baie des Chaleurs et sur le littoral gaspésien se dessine peu à peu. Cependant, la plupart des sources datent du XVIII siècle et elles font référence presque uniquement aux Basques du Labourd en France. Les sources ne disent presque rien de la présence des Basques en provenance des provinces du Gipuzkoa et de la Bizkaia en Espagne, pour-

1. Laurier Turgeon, "Pêches basques du Labourd en Atlantique nord (XVI ${ }^{\mathrm{e}}-\mathrm{XVIII}{ }^{\mathrm{e}}$ siècle): ports, routes et trafics ", Itsas memoria, Revista de estudios maritimos del País Vasco 3 (San Sebastián, 2000); 163-178; Mario Mimeault, Destins de pêcheurs: Les Basques en Nouvelle-France (Québec, Septentrion, 2011), 57-60; Miren Egaña Goya, «Presencia de los pescadores vascos en Canadá, s. XVII. Testimonio de la obras de Samuel de Champlain (1603-1633)», Zainak, Cuadernos de Antropología-Ethnografia 33 (San Sebastián, 2010);375-392.

2. Samuel de Champlain, Euvres, dans Charles-Honoré Laverdière, Euvres de Champlain [1870], réédition en facsimilé, 3 vol. (Montréal, Éditions du Jour, 1973), 1062, 1067-1068, 1085-1087, 1104, 1113, 1117, 1159-1161, 1174, 1315-1323 ; cf. Gabriel Sagard, Histoire du Canada et voyages que les Freres Mineurs Recollects y ont faicts pour la conversion des Infidelles (Paris, Claude Sonnius, 1636), 841-842.

3. Pierre Nadon, La Baie du Grand Pabos: Une seigneurie gaspésienne en Nouvelle-France au XVIII siècle, Archéologiques: Collection mémoires de recherche vol. 1 (Québec, Association des archéologues du Québec, 2004), 18.

4. Mario Mimeault, «L'industrie canadienne de la pêche à la morue (1663-1758); naissance d'une industrie et savoir emprunté », dans Michaël Augeron, Jacques Péret et Thierry Sauzeau, dir., Le Golfe du Saint-Laurent et le Centre-Ouest français. Histoire d'une relation singulière (XVII ${ }^{e}$-XIX ${ }^{e}$ siècle) (Rennes, Presses universitaires de Rennes, 2010), 17-35.

5. Peter Bakker, "The Language of the Coast Tribes is Half Basque": A Basque-American Indian Pidgin in Use between Europeans and Native Americans in North America, ca. 1540-ca. 1640 ”, Anthropological Linguistics, 31 (1989): 117-147. 
tant plus nombreux dans le golfe du Saint-Laurent que ceux du Labourd ${ }^{6}$ et qui sont là du $\mathrm{XVI}^{\mathrm{e}}$ siècle jusqu'au tournant du XVIII ${ }^{\mathrm{e}}$ siècle, ce qui est avéré par les analyses récentes sur la provenance des céramiques retrouvées sur les gisements archéologiques ${ }^{7}$. On peut alors se demander comment expliquer leur absence dans les écrits, et s'ils pêchaient aussi dans la baie des Chaleurs.

Dans ce contexte d'écrits tardifs et vraisemblablement partiels, le routier du pilote Piarres Detcheverry, publié à Bayonne en 1677, apparaît comme une source unique par sa description systématique des routes et lieux fréquentés par les Basques dans la baie des Chaleurs et sur la pointe de la péninsule gaspésienne au XVII ${ }^{e}$ siècle ${ }^{8}$. Hormis ses instructions aux pilotes, ce document singulier contient aussi des indices sur la nature et l'ancienneté de la présence basque dans cette région. Il est toutefois peu connu, d'abord parce qu'il est publié en basque, une langue peu familière à la plupart des historiens s'intéressant à la baie des Chaleurs, mais aussi parce que sa compréhension requiert une connaissance du vocabulaire maritime basque ancien et de l'hydrographie et de la géographie locales. Pourtant, ce routier modifie l'historiographie de la période telle que nous la connaissons, du fait qu'il indique la présence des Basques à des endroits précis dans la baie des Chaleurs à une époque où les autres sources n'en parlent pas.

Nous présentons ici la portion du routier qui concerne la pointe de la péninsule gaspésienne et la baie des Chaleurs, dans le basque d'origine accompagné de notre traduction française (en annexe). Si ce travail de traduction nous a confrontés aux difficultés d'une telle entreprise, dont certaines restent imparfaitement résolues, le résultat apporte néanmoins un éclairage nouveau et souvent original sur la présence basque dans cette région au $\mathrm{XVII}^{\mathrm{e}}$ siècle. Notre but premier est de rendre ce texte disponible aux non-bascophones et de fournir des éléments contextuels sur le routier et les informations qu'il contient. De plus, nous tentons de mieux comprendre pourquoi les autres sources ne révèlent guère ce qui semble être une présence basque significative dans cette région au XVII ${ }^{e}$ siècle. La réponse reste partielle, mais la nationalité espagnole de plusieurs Basques peut avoir contribué à leur faible visibilité dans les écrits français. Enfin,

6. Brad Loewen et Vincent Delmas, "The Basques in the Gulf of St. Lawrence and adjacent shores», Canadian Journal of Archaeology, 36 (2012): 357.

7. Saraí Barreiro Arguëlles, «Ciudadanos del Atlántico. Las redes de aprovisionamiento trasatlánticas de las pescas vascas en Canadá a través de su cerámica, siglos XVI-XVIII ", mémoire de maîtrise (anthropologie), Université de Montréal, 2015.

8. Piarres Detcheverry, Liburu hau da ixasoko nabigacionecoa (Bayonne, Antoine Fauvet, 1677); réédition en facsimilé (San Sebastián, Txertoa, 1985). 
nous examinons l'éclairage nouveau qu'apporte le routier sur la solidarité basque transfrontalière et sur les relations des Basques dans la baie des Chaleurs avec les Amérindiens et avec les autorités coloniales.

\section{L'GUVRE DE PIARRES DETCHEVERRY}

Hormis ce routier, l'œuvre de Detcheverry comporte deux versions légèrement distinctes d'une carte de Terre-Neuve et du golfe du Saint-Laurent en date de 1689. Les cartes portent la légende "faite pour Monsieur [Antoine] Parat Gouverneur de Plaisance » et elles sont caractéristiques de cette "école basque» de cartographie qui connaît un essor entre 1674 et $1713^{9}$. Ces cartographes semblent être issus du milieu des pêcheurs basques, mais les légendes des cartes sont en français; d'ailleurs, le premier d'entre eux, Denis de Rotis, précise que sa carte est "faite à Saint Jean de Luz». Les cartes se distinguent par l'indication des ports fréquentés par les pêcheurs basques, souvent ignorés par les cartographes contemporains français, anglais et hollandais. Entre autres, c'est grâce à cette école que l'on est à même de constater que la côte occidentale de Terre-Neuve était émaillée de toponymes basques et que ce littoral portait alors le nom de "côte basque ${ }^{10}$ ».

L'explication de cette production cartographique est liée à la crainte des Basques de perdre leur droit coutumier de mener la pêche saisonnière, aux mains de la France et de l'Angleterre qui établissent alors leur pouvoir dans la région. En effet, un long texte figurant sur une carte anonyme, le «Plan géométral» de 1713, décrit l'ancienneté des pêches basques à Terre-Neuve et fait valoir les droits coutumiers qui en découlent ${ }^{11}$. Au cours du XVII ${ }^{\mathrm{e}}$ siècle, les Basques adoptent plusieurs stratégies pour protéger leur économie maritime, dans un monde perturbé par les guerres et la course. On voit une concertation entre les provinces basques des deux côtés de la frontière pyrénéenne à travers une suite de "traités de Bonne Correspondance» de $1653,1675,1688$ et 1694, qui visent à assurer la navigation basque en période d'hostilités franco-espagnoles ${ }^{12}$.

9. Miren Egaña Goya, «"Le plan géométral de l'île de Terreneuve”. L'école basque de cartographie», Zainak, Cuadernos de Antropologia-Ethnografia, 21 (2002): 253-267.

10. Miren Egaña Goya, «Basque toponymy in Canada», Onomastica canadiana, 74, 2 (1992): 53-74; "Les toponymes basques au Québec», Le Naturaliste canadien, 119, 1 (1995): 54-57; Selma (Huxley) Barkham, The Basque Coast of Newfoundland (Plum Point, The Great Northern Peninsula Development Corporation, 1989).

11. M. Egaña Goya, «Le plan géométral...», op. cit.

12. Caroline Lugat, "Les traités de "Bonne Correspondance" entre les trois provinces maritimes basques (XVI ${ }^{e}$-XVII ${ }^{e}$ siècles)", Revue Historique, 304, 3 (2002): 611-655, et «Les Traités de Bonne Correspondance: une dérogation aux règles de droit maritime international? (XVI ${ }^{e}$-XVIII ${ }^{e}$ siècles)", Itsas 
En outre, en 1692 et 1697, dans le but d'articuler une stratégie diplomatique pour protéger les droits coutumiers des Basques à Terre-Neuve, l'Amirauté espagnole tient des audiences à San Sebastián auprès des pêcheurs transatlantiques. Plusieurs capitaines témoignent que les Français ne les ont jamais dérangés dans la poursuite de leur pêche. La stratégie diplomatique tombe à court d'arguments, cependant, car le traité d'Utrecht de 1713 exclut les Gipuzkoans de Terre-Neuve et sonne le glas à leur pêche transatlantique vieille de deux siècles ${ }^{13}$.

C'est donc dans ce contexte d'hostilités franco-espagnoles endémiques, de solidarité transfrontalière des Basques et de leurs contacts grandissants mais défensifs avec les autorités coloniales que s'inscrit le routier de Piarres Detcheverry. D’ailleurs, l'auteur publie son livre après la guerre de Hollande quand les marins basques se trouvent de nouveau pris au centre d'un différend géopolitique majeur. C'est peut-être pourquoi Detcheverry le rédige en basque, une langue neutre qui protégera les capitaines si leur navire se fait arraisonner par l'ennemi.

Si le contexte des pêches basques est de mieux en mieux connu, on sait peu de choses sur l'auteur du routier et des deux cartes, Piarres Detcheverry dit Dorre (ou Dorré). Par exemple, on ne sait rien des campagnes qu'il aurait menées en Amérique. On sait seulement qu'il venait d'une famille de marins et d'armateurs de Saint-Jean-de-Luz, où il vivait toujours en 1690 , alors âgé de 53 ans ${ }^{14}$. On peut alors situer sa naissance en 1636 ou 1637 et établir son âge à environ 40 ans au moment de la parution de son livre. Il appert que le routier est l'œuvre d'un marin accompli, ce qui renforce l'autorité d'un document qui allait conduire son auteur, douze ans plus tard, à se voir confier le mandat de dresser une carte générale de Terre-Neuve et du golfe du Saint-Laurent. La réputation de Detcheverry était telle que son routier fut le premier livre de type professionnel publié en langue basque, les publications dans cette langue étant jusqu'alors limitées à des livres à caractère religieux ${ }^{15}$.

Quant au document lui-même, considérant ses difficultés linguistiques et techniques, nombre de chercheurs se sont arrêtés à la page titre, où

Memoria. Revista de Estudios Marítimos del País Vasco, 5 (2006): 301-308; Álvaro Aragón Ruano et Xabier Alberdi Lonbide, «Relaciones comerciales entre puertos franceses y guipuzcoanos en las décadas finales del siglo XVI y la primera mitad del siglo XVII; síntoma de vitalidad económica », Pedralbes, 27 (2007): 219-262 ; L. Turgeon, "Pêches basques du Labourd... », op. cit., 174-175.

13. Enrique de Gandía, Primitivos navegantes vascos (Buenos Aires, Ekin, 1942), 41-62.

14. Aurélie Arcocha-Scarcia, "Itsassoco biayetaco othoicen araldea, "La série de prières pour les voyages en mer” de Joannes Etcheberri de Ciboure (1627)", Lapurdum, 4 (Bayonne, 1999): 9-44.

15. Luis Michelena, Historia de la literatura vasca (Madrid, Minotauro, 1960), 78; Robert Lawrence Trask, A History of Basque (Londres, Routledge, 1996), 47-48. 
l'auteur annonce volontairement qu'il a «mis en basque» un livre plus ancien d'instructions aux pilotes, publié en français à Bordeaux en 1579, et réédité plusieurs fois jusqu'en 1667. Il s'agit là d'une référence au routier de Martin de Hoyarsabal, un navigateur de Ciboure, le port voisin de Saint-Jean-de-Luz ${ }^{16}$ :

Ce livre est celui de la navigation de la mer. Fait en français par Martin de Hoyarzabal. Et mis en basque par Piarres Detcheverry ou Dorre, et quelque plus avancé. À Bayonne, [...] dans l'imprimerie d'Antoine Fauvet à côté du couvent des Carmes, $677[\text { [sic }]^{17}$.

La modestie de l'auteur, ou son astuce, en se présentant comme un simple traducteur est démentie par les nombreux aspects originaux du routier qui apparaissent à la lecture comparée des deux textes. Comme son prédécesseur, Detcheverry décrit d'abord les côtes euro-atlantiques. Il s'en tient à l'organisation générale du livre de Hoyarsabal, en traitant des routes côtières autour de la péninsule ibérique à partir du Pays Basque et jusqu'à Cadix et Alicante, puis des routes du Pays Basque à la Flandre et aux îles Britanniques. Si Detcheverry se permet quelques remaniements du texte de Hoyarsabal, il reste dans la tradition des routiers connus depuis le XIV siècle, où les connaissances maritimes s'enrichissent et s'affinent progressivement et parfois quasi imperceptiblement d'un ouvrage à l'autre ${ }^{18}$. S' inscrire ainsi dans l'ancien savoir était, semble-t-il, un gage de probité aux yeux de la confrérie prudente des pilotes de mer.

Il aurait donc été normal pour Detcheverry de s'appuyer aussi sur le livre de Hoyarsabal dans sa description des routes de Terre-Neuve, qui occupe les derniers chapitres du routier. Ce n'est toutefois pas le cas. Bien au contraire, Detcheverry couvre d'autres régions géographiques que son prédécesseur et il adopte une voix narrative qui lui est propre. Rappelons que Hoyarsabal décrit principalement la côte atlantique de Terre-Neuve, de la baie de Plaisance au détroit de Belle-Île, dans un texte qui couvre 18

16. Martin de Hoyarsabal, Voyages avantureux (Bordeaux, Guillaume Millanges, 1579); $c f$. Charles de La Roncière, "Le premier routier pilote de Terre-Neuve (1579)", Bibliothèque de l'École des chartes, 65 (1904): 116-125; Michael M. Barkham, «New Documents Concerning the French Basque Pilot Martin de Hoyarsabal, Author of the First Detailed Rutter for the "New Found Land" (1579)", Newfoundland Studies, 19 (2003): 103-131.

17. P. Detcheverry, Liburu..., op. cit.; «Liburu hau da ixasoco nabigacionecoa. Martin de Hoyarzabalec egiña Francezes. Eta Piarres Detcheverry, edo Dorrec escararat emana, eta cerbait guehiago abançatuba. Bayonan, [...] Fauvet imprimerian Carmesseteco aldean, DC.LXXVII.»

18. Albrecht Sauer, Das "Seebuch": Das alteste erhaltene Seehandbuch und die spatmittelalterliche Navigation in Nordwesteuropa, Schriften des Deutschen Schiffahrtsmuseums (Hambourg, Kabel, 1996), 106-107, 225-227. 
pages et compte environ 4800 mots $^{19}$. Detcheverry, pour sa part, consacre non moins de 33 pages et 18500 mots à sa description des routes du Nouveau Monde ${ }^{20}$. Il a d'ailleurs supprimé l'organisation à la façon des routiers médiévaux du livre de Hoyarsabal et enrichi son propre texte de détails contextuels qui en font une source historique plus féconde. Et surtout, il s'intéresse à de nouvelles régions. S'il suit Hoyarsabal en longeant la côte est de Terre-Neuve et le détroit de Belle-Île (p. 104-117), il entreprend à son propre compte la description des côtes sud et ouest de Terre-Neuve et des routes de la péninsule gaspésienne et de la baie des Chaleurs (p. 118-136). Ce faisant, il offre la première description systématique de ces régions après Jacques Cartier en 1534.

$S$ 'inscrivant dans une longue tradition de navigation basque vers TerreNeuve et le golfe du Saint-Laurent, Detcheverry s'appuie sur les connaissances héritées du livre de Hoyarsabal, mais innove aussi par une organisation plus moderne et l'inclusion de nouvelles routes à l'intérieur du Golfe. La spécificité basque de son routier est aussi plus affirmée, car il s'adresse aux marins basques dans leur langue. De plus, les nouvelles régions qu'il couvre semblent être fréquentées surtout par les pêcheurs basques migratoires ${ }^{21}$ à cette époque. Alors que les sources françaises traitent souvent des Basques selon un regard externe, comme elles représentent aussi les Autochtones, Detcheverry les positionne plutôt comme les protagonistes de leur propre histoire, ouvrant alors une perspective interne privilégiée sur l'espace des pêcheurs basques vers 1677. En outre, le pilote basque semble à son tour ausculter les Français d'un point de vue externe. Ce jeu de regards croisés révèle un paysage de dynamiques culturelles et enrichit notre compréhension des sources françaises pour la baie des Chaleurs et l'extrémité de la péninsule gaspésienne.

\section{L'ITINÉRAIRE DU PILOTE. ROUTES, MOUILLAGES, PAYSAGE MARITIME}

Afin de suivre les instructions du routier, on doit évidemment connaitre les unités de distance et le système cardinal que l'auteur utilise. Comme principale unité de distance, Detcheverry emploie la lieue de 2 milles marins $(3,70 \mathrm{~km})$. C'est aussi la lieue utilisée par Hoyarsabal, tandis que d'autres navigateurs, comme Jean Fonteneau dit Alfonse dit Saintongeais et Cristobal de Barrós au XVI $\mathrm{XI}^{\mathrm{e}}$ siècle, utilisaient plutôt la lieue de 3 milles

19. M. de Hoyarsabal, Voyages avantureux..., op. cit., 103-120.

20. P. Detcheverry, Liburu..., op. cit., 104-136.

21. Les pêcheurs appelés "migratoires», "saisonniers» ou "transatlantiques» vivaient en Europe et pêchaient pendant l'été en Amérique du Nord à des stations côtières qu'ils n'occupaient pas à l'hiver. 
marins $(5,55 \mathrm{~km})$ correspondant à une minute de latitude ${ }^{22}$. Parfois, Detcheverry emploie d'autres unités pour des distances plus courtes, comme l'agusta dans la portion du routier que nous présentons ici. Imprécises et peu connues aujourd'hui, de telles mesures étaient autrefois familières aux marins ${ }^{23}$. Pour indiquer les caps à suivre, Detcheverry utilise le nord magnétique qui, à son époque, se déclinait d'environ 15 degrés à l'ouest du nord polaire dans la baie des Chaleurs. Enfin, le routier donne les profondeurs d'eau en brasses $(1,95 \mathrm{~m})$.

Ayant traversé le golfe du Saint-Laurent à partir du sud de Terre-Neuve, Detcheverry commence sa description à la pointe de Caispe (cap Gaspé), où il trouve ses repères et se dirige aussitôt vers la pointe de Forcemolue (pointe Saint-Pierre), trois lieues au sud. Sa destination est un poste de pêche situé à l'intérieur de la baie de Forcemolue, à l'endroit appelé aujourd'hui Belle-Anse ${ }^{24}$. Il y mentionne la grave et le mouillage des navires ainsi que la présence d'un quai. Puis, il reprend le chemin en longeant la côte jusqu'à un endroit qu'il identifie par le nom de Fundamen. Ce toponyme, qui signifie «fondation» en latin, ne peut faire autrement que nous interpeller. À première vue, il semble désigner l'établissement seigneurial de la Petite-Rivière, fondé à Barachois en 1672 par Pierre Denys de la Ronde ${ }^{25}$. Le Récollet Chrestien Le Clercq en 1675 situe «l’Habitation de monsieur Denys [...] sur le bord d'un bassin vulgairement appelé la Petite-Rivière, séparé de la mer par une belle langue de terre ${ }^{26}$ ».

Sur ses cartes de 1689, Detcheverry positionne ce lieu au fond de la baie de Forcemolue et l'identifie par la légende «habitacion» sur une carte et le toponyme «Barrachoa» sur l'autre (figures 1 et 2). Si l'on ne trouve que le toponyme Fundamen dans son routier, ses indications conduisent bien au lieu actuel de Barachois. En partant de l'anse de Forcemolue (BelleAnse), Detcheverry s'adresse au capitaine en lui faisant longer la côte jusqu'à une pointe de sable qui est sa destination. À ce moment, le capi-

22. Jean Alfonce, Les voyages aventureux (Poitiers, Pélican, 1559); Cristobal de Barrós (1571), Archivo general de Simancas (Espagne), GA 75 / 24, cité dans Selma (Huxley) Barkham, «Los Vascos y las pescerías transatlánticas, 1517-1713 », p. 58, note 10, dans Enrico Ayerbe et Selma (Huxley) Barkham, dir., Itsasoa 3. Los Vascos en el marco Atlántico Norte, Siglos XVI y XVII (San Sebastián, ETOR, 1988), 26-164.

23. A. Sauer, Das "Seebuch"..., op. cit., 142-151, 222-223 (résumé en français).

24. Sur l'hydronyme «baie de Forcemolue», voir Jean Poirier, «Exemple de diachronie choronymique - Le vocable La Malbaie en Gaspésie», Revue internationale d’onomastique, 2 (1973): 115-121.

25. Cf. Marie-Claude Francoeur, «Le développement socio-économique des seigneuries gaspésiennes sous le Régime français; un modèle régional unique », mémoire de maîtrise (histoire), Université Laval, 2008, 37-41, 64-68; R. P. [Révérend Père] Hugolin [baptisé Stanislas Lemay], L'établissement des Récollets à l'Isle Percée 1673-1690 (Québec, sans éditeur, 1912), 14.

26. Chrestien Le Clercq, Nouvelle relation de la Gaspésie (Paris, Amable Auroy, 1691), 22. 
taine doit immobiliser son navire et expédier une chaloupe pour repérer la pointe et trouver la bouée qui balise l'entrée du port. Une fois remis en route, le navire laissera la bouée à tribord, du côté de la terre. Puis, il tournera à bâbord où le capitaine pourra mouiller à l'ancre ou amarrer à terre, selon les câbles qu'il possède; la baie sera alors au nord-ouest et le capitaine y trouvera tout ce dont il a besoin pour sa "commodité», ce qui laisse entrevoir l'existence d'infrastructures à cet endroit.

Il nous reste à examiner pourquoi Detcheverry nomme ce lieu Fundamen dans son routier, un nom dont la portée symbolique contraste avec le caractère plus descriptif des toponymes qu'il utilise sur ses cartes. Selon le Diccionnario General Vasco, Fundamen est calqué du latin et porte les sens de "fondement, base ${ }^{27}$ ». Selon une perspective coloniale française, ce toponyme semble renvoyer à la création en 1672 de la seigneurie de l'île Percée, concédée à Pierre Denys de la Ronde qui établit un poste et une mission à la Petite-Rivière ${ }^{28}$. Vu de la perspective des pêches migratoires basques toutefois, Fundamen acquiert une polysémie historique plus complexe. À plusieurs moments au XVII ${ }^{\mathrm{e}}$ siècle, les Basques font valoir leur droit ancien de pêcher et de commercer dans le territoire réclamé par la France autour du golfe du Saint-Laurent ${ }^{29}$. Ils assoient ce droit sur les «us et coutumes» de la mer et en particulier ceux de «la route de Terre-Neuve ${ }^{30}$ », et ils insistent sur l'antériorité de leur présence par rapport aux Français et aux Anglais.

Cette idée de la primauté des pêches basques, que l'on voit déjà chez Marc Lescarbot, se précise en 1647 lorsque le juriste bordelais Étienne de Cleirac affirme que les «Basques de Guyenne» ont trouvé Terre-Neuve et les terres de "Canada ou Nouvelle-France» cent ans avant les navigations de Christophe Colomb ${ }^{31}$. La source de cette information reste obscure, toutefois, car les archives mentionnent un voyage basque à Terre-

27. Orotariko Euskal Hiztegia (Diccionnario General Vasco) 2014 Académie de la Langue Basque, Bilbao, "fundamen » www.euskaltzaindia.net $/$ index.php?option=com_content $\&$ view $=$ article $\&$ id $=2768$ Itemi $\mathrm{d}=413 \&$ lang=eu [consulté le 2 janvier 2015].

28. R. P. Hugolin, L'établissement des Récollets..., op. cit., 10-12.

29. René Bélanger, Les Basques dans l'estuaire du Saint-Laurent (Montréal, Les Presses de l'Université du Québec, 1971).

30. Brad Loewen, «Les barriques de Red Bay et l'espace atlantique, vers 1565 », thèse de doctorat (histoire), Université Laval, 1999, 235-241.

31. Étienne Cleirac, Us et coustumes de la mer (Bordeaux, Guillaume Millanges, 1647), 151; «... [les Basques de Guyenne] ont découvert cent ans avant les navigations de Christophle Colomb, le grand $\&$ le petit banc des morues, les terres de Terreneufve, de Capberton \& Baccaleos (qui est à dire Morue en leur langue), le Canada ou nouvelle France...». D’autres auteurs ont ensuite repris la date de 1392 (ou parfois 1372). Brad Loewen, «Historical Data on the Impact of 16th-Century Basque Whaling on Right and Bowhead Whales in the Western North Atlantic ", Canadian Zooarchaeology, 26 (2009): 13. 
Figure I

Carte de Piarres Detcheverry, 1689 (version I). Détail de la Gaspésie, de la baie des Chaleurs et de l'ile du Prince-Édouard.

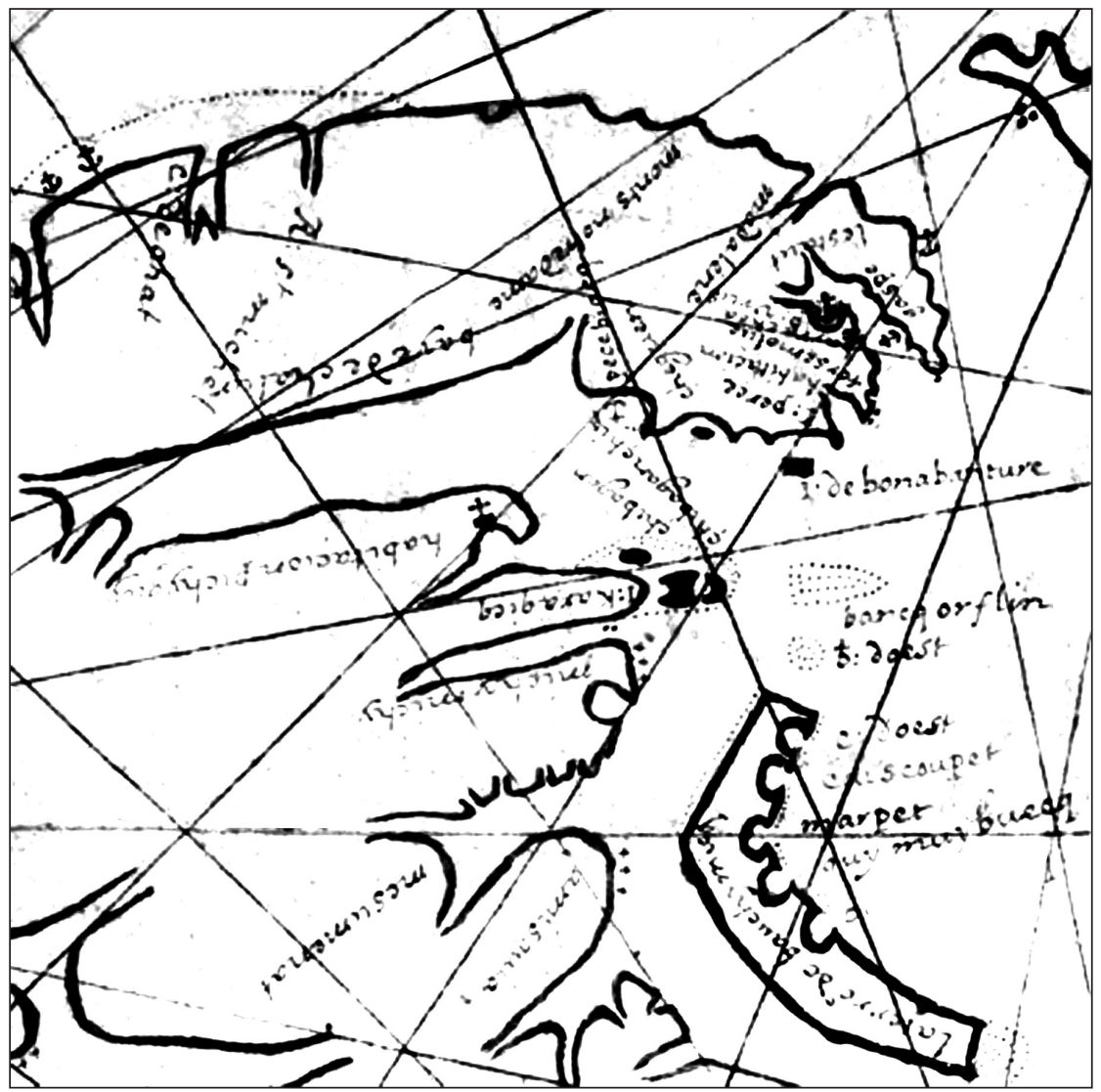

Figure 2

Carte de Piarres Detcheverry, 1689 (version 2).

Détail de la Gaspésie et de la baie des Chaleurs. $\Rightarrow$ 


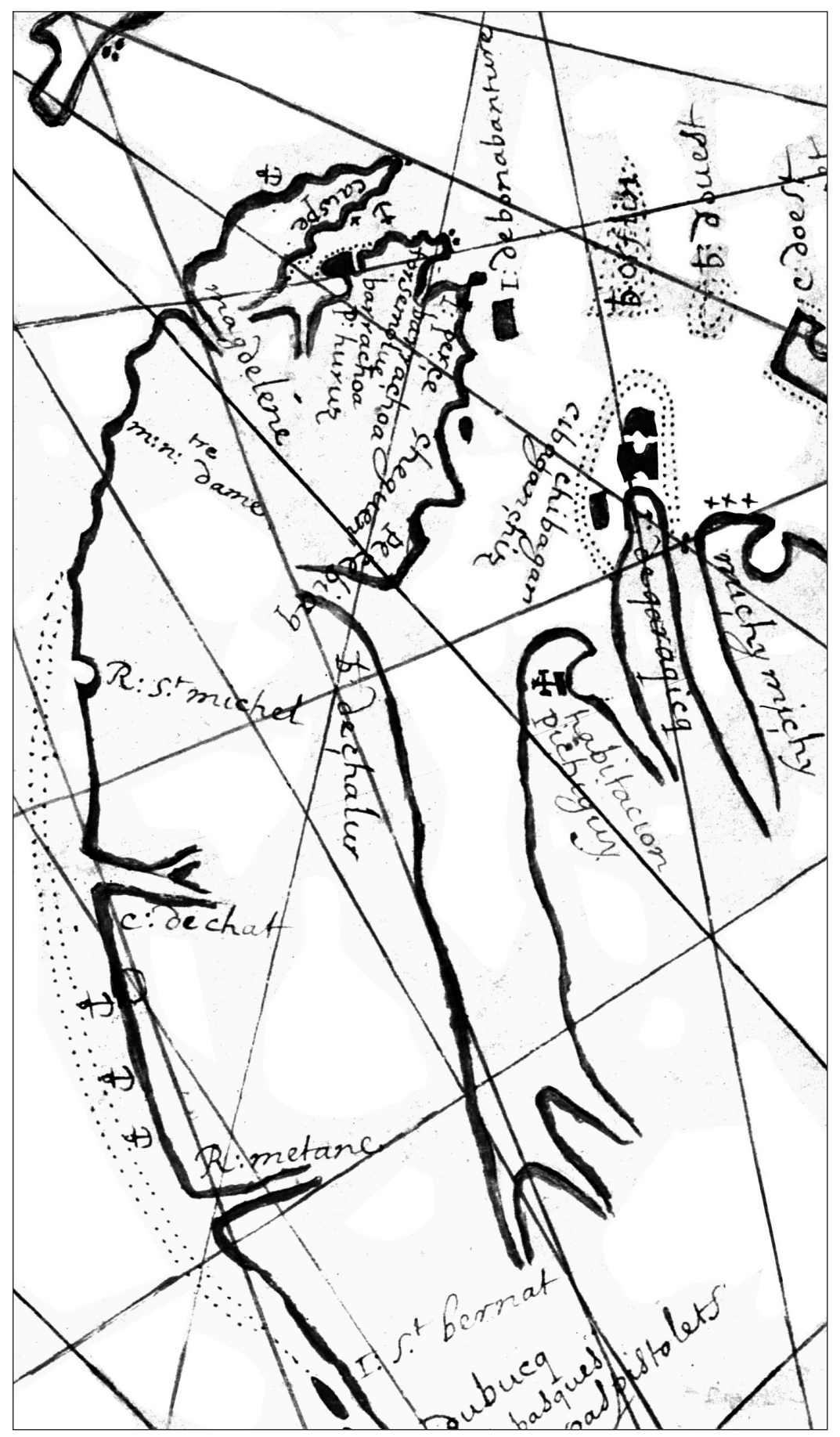


Neuve pour la première fois en $1517^{32}$. Il n'empêche que plusieurs écrits du XVII ${ }^{e}$ siècle affirment l'antériorité des pêches basques et en font un argument en faveur des droits des pêcheurs migratoires basques en territoire colonial français et anglais. Or, sur le littoral gaspésien, la création de seigneuries de pêche sédentaire et le décret de l'Ordonnance de la Marine de 1681 favorisant les pêcheurs migratoires français ont eu l'effet de marginaliser les pêcheurs basques de l'Espagne ${ }^{33}$. Il en ressort l'image d'un paysage maritime contesté où le toponyme Fundamen pourrait indiquer le lieu de fondation des pêches transatlantiques ou rappeler la "grande pescherie» mentionnée par Jean Fonteneau dit Alfonse dans cette région vers $1545^{34}$.

Alors que Detcheverry s'attarde à la description de Fundamen, c'est à peine s'il mentionne les alentours de Percé $e^{35}$. Il ne fait qu'utiliser l'île Bonaventure comme repère pour entamer la traversée de la baie des Chaleurs vers Chegoanchis (Miscou), dont le havre sera ensuite le point de rayonnement pour d'autres routes dans la baie. Jusqu'à ce point dans le texte, Detcheverry s'adresse aux capitaines de navire, mais à partir de Miscou, il écrit aux maîtres de chaloupe et de charroi, des embarcations utilisées pour la pêche. Il expédie une chaloupe à Karakik (Caraquet), via un chenal en forme de " $Z$ » qui s'amorce vis-à-vis de l'île Pokesudie et conduit entre la terre ferme et l'île Caraquet dont la côte sud forme une anse protégée. Ensuite, Detcheverry trouve ses repères dans les montagnes au nord de la baie des Chaleurs pour aller en droite route à Paspébiac. Puis il longe la côte jusqu'à Chequen, où il dit que les pêcheurs de Paspébiac sèchent leurs poissons. Detcheverry situe Chequen au nord de Miscou, une position confirmée par ses cartes et qui correspond à PortDaniel, même si Nicolas Denys écrit en 1672 que les pêcheurs de Paspébiac sèchent leurs prises «au petit Paspec-biac», soit Bonaventure ${ }^{36}$.

32. Jacques Bernard, «Les débuts de la pêche à Terre-Neuve, vus de Bordeaux (1517-1550). Bilan et perspective", dans L'Atlantique et ses rivages (1500-1800). Actes du Colloque de 1983, Association des Historiens modernistes des Universités (Bordeaux, Presses universitaires de Bordeaux, 1984), 11-50.

33. L’Ordonnance de la Marine de 1681 réserve la côte entre les caps de Rosiers et d'Espoir aux navires français (voir plus loin). Charles de La Morandière, Histoire de la pêche française de la morue dans l'Amérique septentrionale, vol. 2 (Paris, Maisonneuve et Larose, 1962), 602. Dans les années 1730, les seigneurs canadiens s'opposeront à ce droit, en favorisant une pêche sédentaire. M.-C. Francœur, «Le développement...", op. cit., 90-94.

34. Georges Musset, La Cosmographie, avec l'espère et régime du soleil et du nord, par Jean Fonteneau dit Alfonse de Saintonge (1549) (Paris, Ernest Leroux, 1904), 485-487.

35. M.-C. Francœur, «Le développement... », op. cit., 37-41.

36. Nicolas Denys, Description géographique et historique des Costes de l'Amérique septentrionale (Paris, Louis Billaine, 1672), 214-215; cf. Emmanuel Jumeau, La grande baye de St. Laurens en la Nouvelle France 
Revenant à Paspébiac, Detcheverry décrit ensuite la route vers un lieu qu'il appelle Estancia ou Pecheguy. Il s'agit de Népissiguit où, dans les années 1670, il existait une mission et une ferme exploitée par Philippe Esnaut, sieur de Barbocan, pour le compte de Nicolas Denys ${ }^{37}$. Detcheverry laisse entendre, en disant "si par hasard tu dois y aller», que ce lieu n'était pas une destination usuelle des pilotes basques. Alors que Pecheguy est sans doute le même mot que Népissiguit, estancia signifie, dans le contexte colonial espagnol du XVII ${ }^{\mathrm{e}}$ siècle, soit une reducción (un établissement d'Autochtones domiciliés) soit une hacienda (un domaine rural). Detcheverry indique ce lieu sur ses cartes avec une croix ${ }^{38}$ et la légende "habitaçion Pichiguy ${ }^{39}$ ». L'ensemble de ces toponymes évoque à la fois une mission, une résidence coloniale et une présence autochtone. On obtient alors une idée complexe de l'établissement et de ses résidants, bien que Detcheverry ne fasse pas référence au seigneur français et dise simplement qu'on allait arriver "chez les gens", sans doute les employés d'Esnaut ou des Autochtones rattachés à ce lieu. Cette façon oblique de représenter Estancia, à l'instar de Fundamen, détonne par rapport au reste du paysage des pêcheurs basques. Telles des tours de guet, les deux établissements français marquent les frontières de l'enclave basque dans la baie des Chaleurs.

Avant de clore son chapitre sur la baie des Chaleurs, Detcheverry s'adresse de nouveau aux pilotes des navires qui veulent se déplacer de Miscou à Caraquet. À Miscou même, il décrit les lieux de mouillage et d'amarrage dans la rade, en mentionnant la présence d'un quai et de vigneaux pour sécher la morue. Il dit que jadis l’entrée était balisée et il déplore la perte de cette pratique qui aidait les pilotes apprentis. Le navigateur termine ses instructions avec les approches et le mouillage à préconiser par les grands navires arrivant à Paspébiac.

(1685), reproduit dans W. F. Ganong, dir., New Relation of Gaspesia... by Father Chrestien Le Clercq (Toronto, The Champlain Society, 1910), 11.

37. Georges MacBeath, «Esnault de Barbaucannes (Barbocant), Philippe», Dictionnaire biographique du Canada en ligne; www.biographi.ca/fr/bio/enault_de_barbaucannes_philippe_2F.html [consulté le 2 janvier 2015]. Réal Ouellet, Chrestien Le Clercq. Nouvelle relation de la Gaspésie - Édition critique (Montréal, Presses de l’Université de Montréal, 1999); 360.

38. La croix renvoie possiblement à la mission de Sainte-Croix à Miramichi. Ce nom rappelait la croix que les Amérindiens utilisaient comme symbole clanique avant l'arrivée des Récollets selon C. Le Clercq, Nouvelle relation..., op. cit., 172-198. Or, sur sa carte de 1685, le Récollet E. Jumeau, La grande baye de St. Laurens..., op. cit., place plusieurs croix à Miramichi avec la légende «la nation de la croix que les sauvages ont reçuë du ciel». Les Récollets prétendaient que ce symbole existait naturellement chez ces Amérindiens et les prédestinait à la foi chrétienne.

39. Nicolas Denys distingue les stations de pêche et les «habitations» au regard de la traite avec les autochtones. N. Denys, Description géographique..., op. cit., tome 2, 468-471. 
Figure 3

L'extrémité de la péninsule gaspésienne, montrant les routes et les lieux indiqués par Detcheverry dans son routier et sur ses cartes.

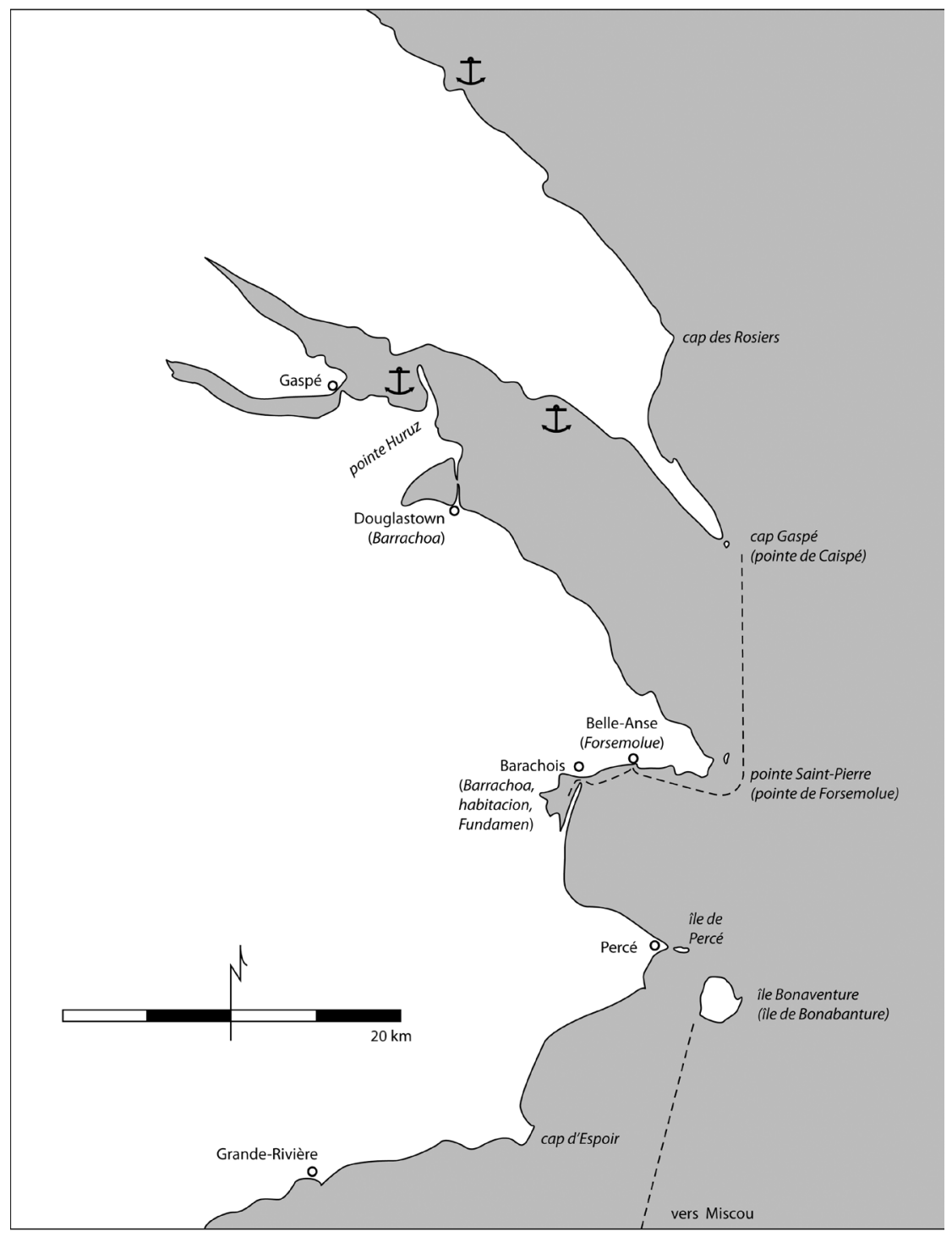


Figure 4

La baie des Chaleurs, montrant les routes et les lieux indiqués par Detcheverry dans son routier et sur ses cartes.

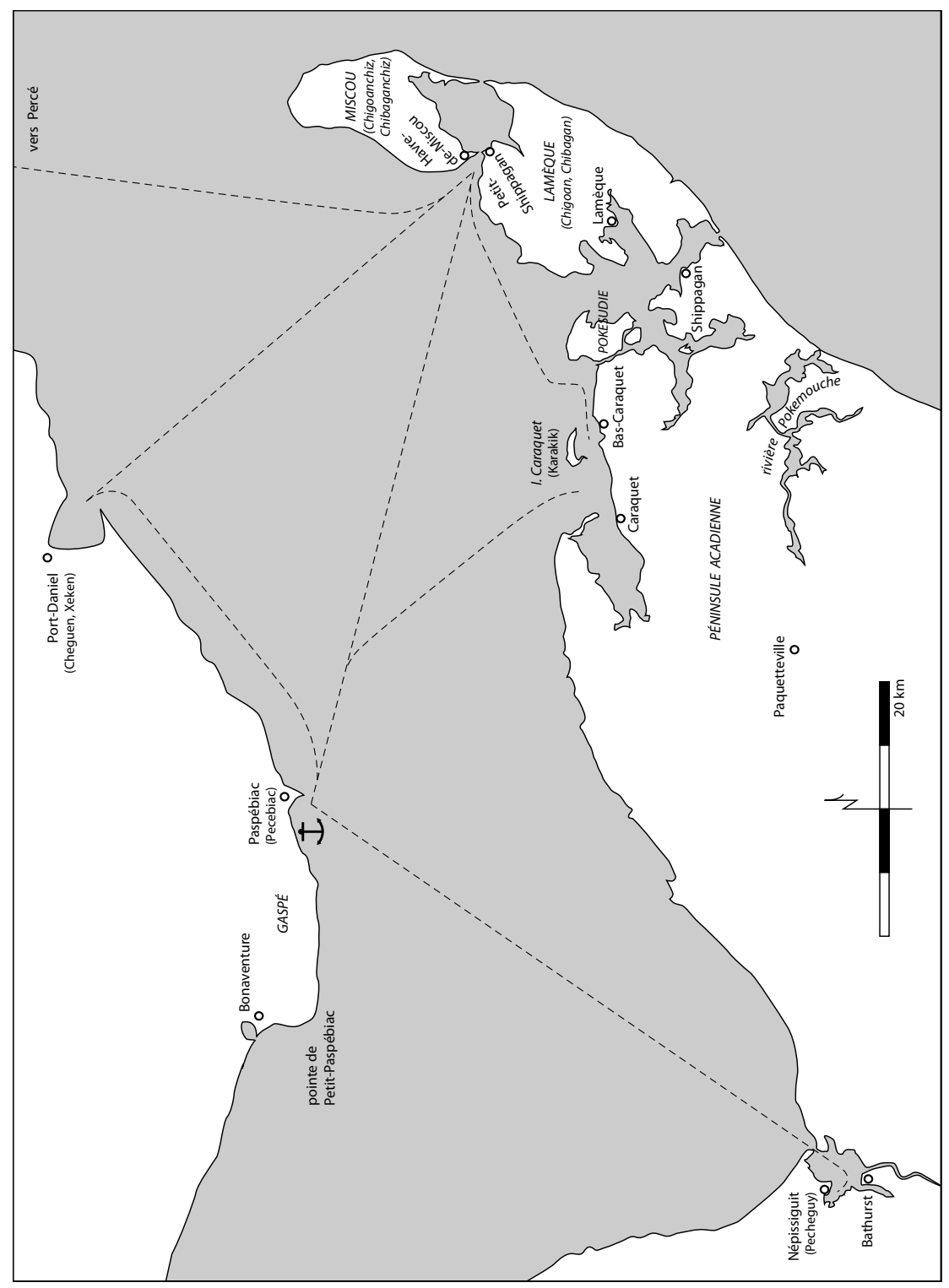


Chacun des endroits mentionnés par Detcheverry acquiert, par le fait même d'être inclus dans le routier, une association avec les pêcheurs basques de la seconde moitié du XVII ${ }^{e}$ siècle. Ce ne sont pas seulement un ensemble de lieux géographiques, mais un paysage culturel tissé par les activités de navigation et de pêche. On voit la navigation des grands navires et des chaloupes côtières, et on aperçoit des aspects des stations de pêche. Detcheverry apporte une perspective basque sur des lieux autrement connus seulement à travers les sources françaises, en particulier Fundamen (Barachois) et Estancia (Népissiguit) qui étaient les points d'appui coloniaux dans la région. Sur la péninsule gaspésienne, son routier identifie Forcemolue ainsi que Fundamen comme des lieux de pêche. Enfin, le routier permet de voir que Miscou, l'île Caraquet et Paspébiac formaient le noyau du paysage culturel maritime basque dans la baie des Chaleurs.

\section{RAPPORTS AUTOCHTONES ET COLONIAUX FRANÇAIS}

Le routier omet de mentionner quelques toponymes qui figurent cependant sur les deux cartes de Detcheverry en date de 1689. C'est le cas de la pointe Huruz dans la baie de Gaspé, ainsi que Percé et quatre noms amérindiens sur l'île du Prince-Édouard. Le corpus de toponymes tirés des cartes et du routier nous renseigne sur les rapports entre les Basques et leurs vis-à-vis français et autochtones. Constatons d'abord qu'un petit nombre de toponymes de Detcheverry ne figurent pas dans d'autres sources contemporaines. C'est le cas seulement de la pointe Huruz $\left(\right.$ Heureuse $^{40}$ ), de Fundamen, Cheguen et Estancia, qui semblent être des contributions uniques de Detcheverry. Plus généralement, Detcheverry utilise des toponymes déjà établis, et en aucun cas il ne prétend baptiser lui-même un lieu. Cependant, à la différence d'autres régions du golfe du Saint-Laurent où les toponymes basques sont fréquents ${ }^{41}$, dans la péninsule gaspésienne et la baie des Chaleurs, les seuls toponymes basques sont Fundamen et Barrachoa. Ce dernier nom, qui plus est, renvoie à un élément géographique (barachois) qui est souvent utilisé dans la toponymie basque autour du golfe du Saint-Laurent et ne constitue alors pas un nom de lieu distinct. L'apport basque à la toponymie de la région est donc relativement faible.

40. Office Public de la Langue Basque, Nola Erran, dictionnaire électronique français-basque, «heureux ", http:/ / nolaerran.org, [consulté le 2 janvier 2015].

41. M. Egaña Goya, "Basque toponymy...», op. cit.; Christian Weyers, "Basque Traces in the Toponymy of Newfoundland and Various Coasts of Atlantic Canada ", Proceedings of the 23rd International Congress of Onomastic Sciences (Toronto, York University, 2009): 1051-1063 ; B. Loewen et V. Delmas, «The Basques...", op. cit. 
La plupart des toponymes utilisés par Detcheverry sont en langue autochtone, une préférence qui est propre à cette région dans la toponymie basque du Golfe. Le routier nomme Chegoanchis, Karakik, Paspébiac, Cheguen et Pecheguy, alors que les cartes ajoutent Chegouan ${ }^{42}$ (Lamèque). En outre, les cartes indiquent quatre autres toponymes autochtones dans l'île du Prince-Édouard, elle-même nommée la terre de Bauchymicq; Caiscoupec (Cascumpec), Marpec (Malpèque), Guymuybuec (Petit-Havre) et Bauchimicq (Rustico). Ce sont possiblement les lieux "fortifiés» par les Basques lors des tensions avec les Français en $1623^{43}$. L'emprunt de noms amérindiens pour les lieux de pêche migratoire n'est nulle part plus dense que dans la baie des Chaleurs, signalant la possibilité d'une relation particulière entre la population permanente autochtone et les pêcheurs saisonniers basques ${ }^{44}$, contrairement aux rapports ponctuels entre pêcheurs français et amérindiens que Nicolas Denys décrit ${ }^{45}$.

Autant les noms de lieux autochtones prédominent dans la toponymie de Detcheverry, autant les noms français s'y font rares. On recense tout au plus trois noms français; Percé, Forcemolue et pointe Huruz. D’ailleurs, plusieurs noms utilisés par les Français à cette époque, comme PortDaniel, Petite-Rivière, Miscou et île Saint-Jean, n'entrent pas dans le lexique du pilote basque. Pendant la même période où Detcheverry documente la baie des Chaleurs, deux auteurs français parcourent la même région: Nicolas Denys ${ }^{46}$ et le missionnaire Récollet Chrestien Le Clercq ${ }^{47}$. Pour les deux Français comme pour le Basque, c'est presque comme si l'Autre n'existe pas dans la baie des Chaleurs. Ce n'est qu'à demi-mot que Denys laisse entrevoir une présence basque à Miscou, lorsqu'il déplore l'abandon de ce port aux «ennemies de la France». Cette prédisposition négative surprend d'autant plus que Denys louange ailleurs, comme à Percé semble-t-il, l'industrie des pêcheurs basques ${ }^{48}$. Quant à Le Clercq, il ne mentionne point les Basques dans la baie des Chaleurs. À en croire le Récollet, les pêcheurs basques se trouvaient dans la «baie des Espagnols», au nord-est du golfe du Saint-Laurent. Son circuit missionnaire, qui partait de Percé/Petite-Rivière et desservait Ristigouche,

42. Chegouan est la racine de Chegoanchis ou «Petit-Chegoan», conservé comme Petit-Shippagan.

43. S. de Champlain, Euvres, op. cit., 652.

44. Peter Bakker, "'The Language of the Coast Tribes is Half Basque"... », op. cit.; Gervais Carpin, Histoire d'un mot: l'ethnonyme «Canadien» de 1535 à 1691 (Sillery, Septentrion, 1995), 67-93.

45. N. Denys, Description géographique... op. cit., tome 2, 470-476.

46. N. Denys, Description géographique..., op. cit., 204-237.

47. C. Le Clercq, Nouvelle relation..., op. cit.

48. N. Denys, Description géographique..., op. cit., tome 1, 193, 196-197, 225, et tome 2, 75, 105, 190. 
Népissiguit et Miramichi, ne faisait halte à aucune des stations basques identifiées par Detcheverry ${ }^{49}$.

Ce clair-obscur géographique dans la baie des Chaleurs s'épaissit eu regard de la carte manuscrite de 1685 du Récollet Emmanuel Jumeau, non publiée à l'époque, qui fait figurer une toponymie dense sur toute la région de Gaspé à Miramichi, y compris dans la baie des Chaleurs ${ }^{50}$. On y retrouve sans distinction presque tous les lieux recensés par Detcheverry et Le Clercq, contrastant avec la sélectivité toponymique de ces derniers. C’est enfin un autre Récollet, Sixte Le Tac, qui éclaire le malaise qui sépare les Basques et l'ordre missionnaire en disant que les Récollets furent mandés, déjà en 1622, de répondre par l'évangélisme à "certains Basques qui venoient faire traitte sans congé à la coste de l'Acadie $\&$ qui donnoient de méchantes impressions des François aux Sauvages de ces costes ${ }^{51} »$. Cette opposition de Français et de "certains Basques» renforce la perception d'espaces exclusifs, aux frontières culturelles reconnues, qui surgit du routier de Detcheverry. Seulement de Gaspé à Percé voit-on l'intersection des espaces français et basque. Il s'agit ici sans doute de Basques labourdains, car l'Ordonnance de la Marine de 1681 réserve le littoral gaspésien entre les caps des Rosiers et d'Espoir aux pêcheurs migratoires de la France ${ }^{52}$. Au contraire, la baie des Chaleurs accueille aussi des Basques de l'Espagne, ce qui explique vraisemblablement les réserves qu'expriment Nicolas Denys et des Récollets à leur endroit.

Malgré la persistance de ces solitudes nationales, les capitaines morutiers du Gipuzkoa pouvaient affirmer, lors des audiences tenues par la Marine espagnole en 1692, que les autorités françaises à Terre-Neuve ne les avaient jamais dérangés dans la conduite de leurs pêches ${ }^{53}$. Leurs témoignages nuancent l'idée selon laquelle les Basques à Plaisance n'étaient qu'une source de troubles voire de "sédition ${ }^{54}$ ». À l'heure où le gouvernement colonial étendait son autorité sur les lieux de pêche, à compter des années 1660, il existait un esprit d'entente avec les pêcheurs

49. C. Le Clercq, Nouvelle relation..., op. cit., 453-457.

50. E. Jumeau, La grande baye de St. Laurens... (1685), op. cit.

51. Eugène Réveillaud, Histoire chronologique de la Nouvelle France ou Canada... par le Père Sixte Le Tac, Récollet (Paris, Fischbach, 1888), 119.

52. Mario Mimeault, «Denis Riverin et la Compagnie des pêches sédentaires du Canada; Les difficultés d'implantation d'une industrie de la pêche en Nouvelle-France ", L'Estuaire, 72 (2012): 49-60 et 73 (2013): 39-48.

53. E. de Gandía, Primitivos navegantes vascos, op. cit., 41-62.

54. Nicolas Landry, Plaisance, Terre-Neuve 1650-1713. Une colonie française en Amérique (Québec, Septentrion, 2008), 331. 
Figure 5

Carte d'Emmanuel Jumeau, 1685.

Détail de la Gaspésie, la baie des Chaleurs et Miramichi.

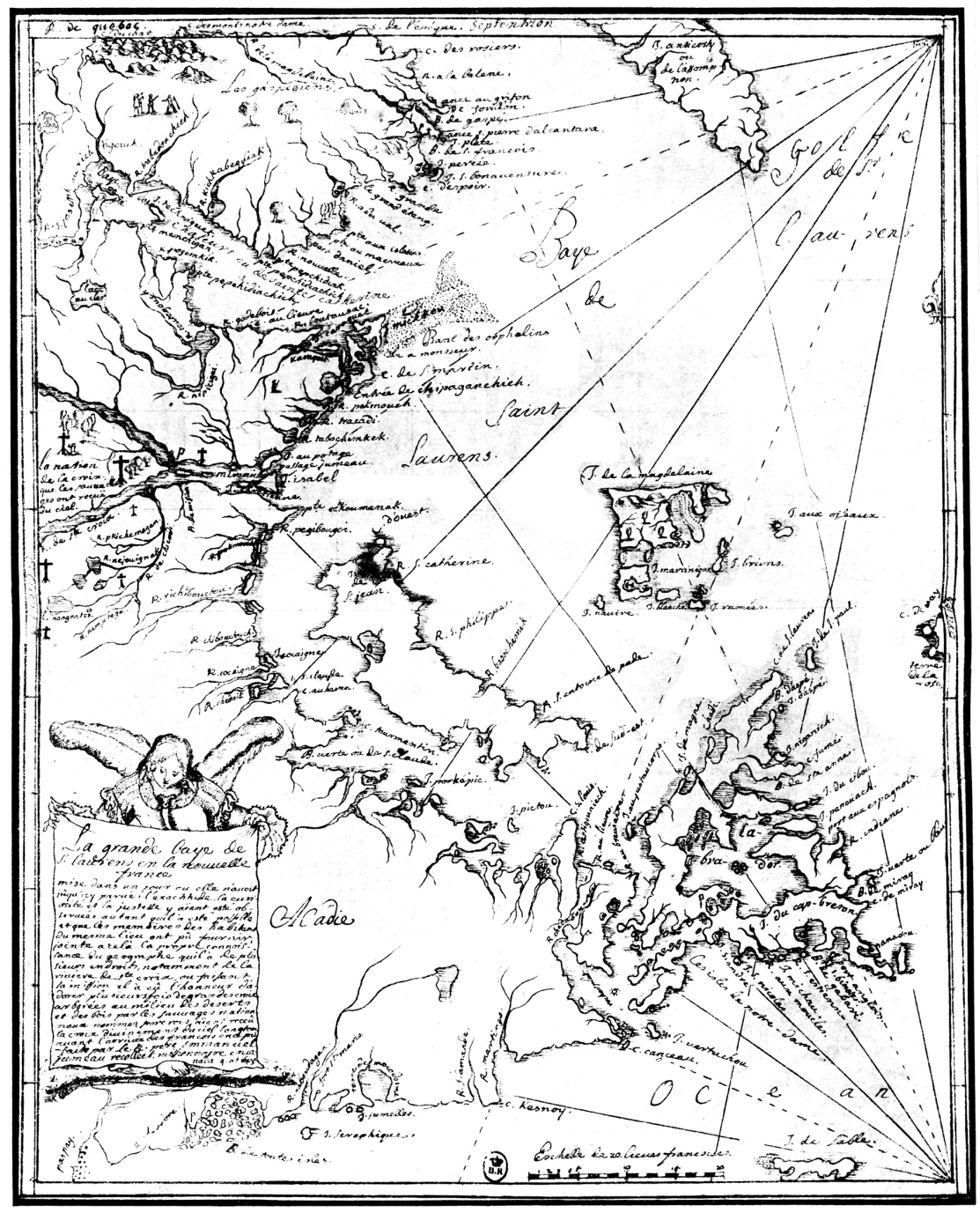

FATHER JUMEAU'S MAP OF 1685 
basques qui contrastait avec la méfiance qui régnait entre les Basques et les autorités françaises durant la première moitié du XVII ${ }^{e}$ siècle ${ }^{55}$.

Au début des années 1620, plusieurs capitaines basques, autant d'Espagne que de France, mènent la pêche et le commerce des fourrures à Miscou et ailleurs dans baie des Chaleurs. En 1623, une crise survient entre eux et Champlain, ce qui conduit les Basques à se replier sur l'île Saint-Jean et à se fortifier. L'intervention de Raymond de la Ralde (ou Larralde de son nom basque), qui agit comme intermédiaire, entraîne la tolérance des Français envers les Basques de 1624 à 1628 et ce commerçant de fourrures à Miscou va s'activer du côté de la France contre les frères Kirke.

En 1631, toutefois, les rapports entre Basques et Français à Miscou se gâtent à nouveau ${ }^{56}$. À compter de 1635 , la guerre de Trente Ans oppose la France et l'Espagne et pendant cette période, les écrits mentionnent très rarement la présence de pêcheurs basques dans le Golfe $\mathrm{f}^{57}$. Sur ces entrefaites, les Français s'installent à Miscou et fondent une colonie sous l'égide de Nicolas Denys et des jésuites ${ }^{58}$. Ils courtisent le chef Juanchou, l'allié des Basques à l'époque de Larralde, en le présentant à la cour de Louis XIII à Paris ${ }^{59}$. La colonie sera toutefois abandonnée en 1643 et la mission jésuite déménagera à Népissiguit, laissant les Basques reprendre leur usage du havre au retour de la paix. Après le traité des Pyrénées en 1659, les épisodes de guerre franco-espagnole sont plus rares et débordent plus rarement outre-Atlantique, ce qui semble favoriser les pêches basques en Nouvelle-France.

Le routier de Piarres Detcheverry révèle donc l'existence d'une enclave basque dans la baie des Chaleurs vers 1677, comprise entre les établissements français à Népissiguit et à Percé/Petite-Rivière. Même si le pilote luzien documente un moment précis dans le temps, la présence basque dans la baie des Chaleurs s'inscrit dans la longue durée du XVII ${ }^{\mathrm{e}}$ au XVIII ${ }^{\mathrm{e}}$ siècle. L'île Caraquet, Paspébiac et surtout Miscou sont les verrous de l'espace basque qui s'étend aussi à Port-Daniel, Percé, Barachois et BelleAnse. Un aspect singulier de ce paysage culturel est l'usage de toponymes autochtones par les Basques, signifiant possiblement la présence de com-

55. R. Bélanger, Les Basques dans l'estuaire du Saint-Laurent, op. cit.

56. S. de Champlain, Euvres, op. cit., 1318-1323; Miren Egaña Goya, «Presencia de los pescadores vascos en Canadá, s. XVII. Testimonio de la obras de Samuel de Champlain (1603-1633)», Zainak, Cuadernos de Antropología-Ethnografia, 33 (2010): 375-392.

57. R. Bélanger, Les Basques dans l'estuaire du Saint-Laurent, op. cit.

58. Narcisse-Eutrope Dionne, "Miscou. Hommes de mer et hommes de dieu ", Le Canada-Français, 2 (1888): 433-447, 521-526.

59. G. Carpin, Histoire d'un mot..., op. cit., 67-93. 
munautés autochtones sur les lieux de pêche, qui entretenaient des liens privilégiés avec les Basques.

Quant aux rapports entre Basques et Français, le silence des uns au sujet des autres par les auteurs de l'époque n'empêchait pas la tolérance des autorités coloniales françaises envers les pêcheurs basques, dont plusieurs venaient de l'Espagne. Seule la guerre franco-espagnole pouvait entamer cette tolérance. Si les Basques de l'Espagne semblent avoir provoqué la méfiance chez Nicolas Denys à Miscou et Louis Jolliet au Labrador ${ }^{60}$, plus le XVII ${ }^{\mathrm{e}}$ siècle avance, plus les pêcheurs gipuzkoans et bizkaians apparaissent comme des alliés indispensables contre l'Angleterre, l'ennemi commun. Dans ce rapport complexe, Piarres Detcheverry a joué un rôle d'intermédiaire à l'instar d'autres Basques d'origine labourdaine, comme Raymond de la Ralde (Larralde) à Miscou dans les années 1620 et François Martel de Brouague (Berhouague) à Brador au XVIII' siècle. Bascophones, mais aussi Français, ils ont servi d'intermédiaires entre les autorités coloniales et les pêcheurs basques retranchés dans leurs enclaves historiques, dans la «baie des Espagnols», sur la "côte basque» de Terre-Neuve, autour de la baie de Plaisance, à Canseau et, non le moindre, dans la baie des Chaleurs.

Pour l'instant, toutefois, aucune source ne permet de tracer une continuité entre la présence basque dans la baie des Chaleurs au XVII ${ }^{e}$ siècle et les références bien connues de Jacques Cartier et de Jean Fonteneau dit Alfonse dit Saintonge, qui révèlent la présence de pêcheurs européens dans cette région dès les années 1530 et 1540. Fonteneau dit qu'une "grande pescherie de molue et de plusieurs aultres poissons" se fait à Anticosti, Gaspé, la baie des Molues et à trois îles dans la baie des Chaleurs, "une grande et deux petites» et dont les principales candidates sont logiquement Bonaventure, Miscou, Lamèque et Caraquet ${ }^{61}$. Cartier semble fournir l'identité de ces pêcheurs précoces lorsqu'il note les mots en espagnol ${ }^{62}\left(\right.$ ou gascon $^{63}$ ) que les Amérindiens lui adressent à Paspébiac

60. Jean Delanglez, Life and Voyages of Louis Jolliet (1645-1700) (Chicago, Institute of Jesuit History, 1948), 210-235.

61. G. Musset, La Cosmographie..., op. cit., 485-487.

62. P. Bakker, "The Language of the Coast Tribes... », op. cit.

63. La langue dans laquelle les Autochtones parlent à Cartier reste problématique, mais leur phrase «napou tou damen asurtar » s'assimile phonétiquement à du gascon défectueux (le subjonctif est absent), n'as páur, tu danam acertar, traduit mot à mot «n'as peur, [nous] te donnons assurer ». Le gascon, parlé de Bordeaux à Bayonne, fut la langue commerciale de certains ports basques. Les Autochtones près du détroit de Belle-Île le parlaient dès 1529; Laurier Turgeon, «Les Français en Nouvelle-Angleterre avant Champlain", dans Raymonde Litalien et Denis Vaugeois, dir., Champlain. La naissance de l'Amérique française (Québec, Septentrion, 2004), 98-112. 
et Port-Daniel, ou encore lorsqu'il utilise le nom espagnol de «cap de Pratto" (prado, pré) pour Percé ${ }^{4}$, et lorsqu'il compare la chaleur du pays à celle de «la terre d'Espaigne». Ailleurs dans le Golfe, il emprunte le nom basque de Port-au-Port (Ophor Portu, port du Vase-à-lait) en l'appelant "cap de Latte» (cap du Lait) ${ }^{65}$. Enfin, dans une figure aussi célèbre qu’ambiguë, il dit que Dieu donna la basse côte nord du Golfe "à Caïn» : peut-il s'agir d'une référence voilée à "Biscaïen", appuyée par la similarité phonétique et la construction figurée de l'Autre? Dans le contexte géopolitique de l'époque, Cartier ne pouvait écrire que les pêcheurs basques de l’Espagne occupaient déjà le territoire qu'il réclamait pour la France, mais il semble avoir trouvé des façons littéraires pour signaler leur présence. Quoi qu'il en soit, les tensions franco-espagnoles entre 1541 et 1579, concentrées autour de la colonie à Cap-Rouge ${ }^{66}$ et des baleiniers dans le détroit de Belle-Île ${ }^{67}$, eurent raison de la navigation française dans le Golfe et possiblement aussi des premières pêches basques dans la baie des Chaleurs. Après le retrait des baleiniers de l'Espagne en 1579, les frères Hoyarsabal de Saint-Jean-de-Luz s'installent près de Tadoussac dès $1581^{68}$, mais le retour des Basques dans la baie des Chaleurs reste non signalé jusqu'au temps de Champlain. Si continuité il y a eue de la présence basque dans cette dernière région entre Cartier et Champlain, la preuve viendra de sources encore à identifier.

\section{CONCLUSION}

Ces brefs commentaires n'épuisent pas la richesse du texte de Detcheverry sur la présence basque en Gaspésie et dans la baie des Chaleurs. En guise de bilan, là où les écrits français parlent seulement de missionnaires et d'Autochtones, Detcheverry révèle un réseau de stations basques, le plus souvent aux noms amérindiens. Le problème singulier soulevé par le routier de Detcheverry concerne le silence des sources françaises par rapport à ces pêcheurs basques. L'analyse interne du routier et la prise en compte d'écrits français donnent à penser que l'enclave basque dans la baie des Chaleurs comportait plusieurs équipages de l'Espagne, tandis que

64. Samuel Eliot Morrison, The Great Explorers; The European Discovery of America (Oxford, Oxford University Press, 1987), 201.

65. M. Egaña Goya, «Basque toponymy in Canada», op. cit.; C. Weyers, «Basque Traces... », op. cit.

66. Bernard Allaire, La rumeur dorée. Roberval et l'Amérique (Montréal, Les Éditions de La Presse, 2013).

67. B. Loewen et V. Delmas, "The Basques...», op. cit.

68. Laurier Turgeon, «Pêcheurs basques du Labourd dans le golfe et l'estuaire du Saint-Laurent au $\mathrm{XVI}^{\mathrm{e}}$ siècle», dans Jean Bourgoin et Jacqueline Carpine-Lancre, dir., L'aventure maritime, du golfe de Gascogne à Terre-Neuve (Paris, Éditions du CTHS, 1995): 213-234. 
les Labourdains s'installaient davantage sur la côte gaspésienne, entre les caps d'Espoir et des Rosiers. C'est semble-t-il le fait que de nombreux Basques soient originaires d'Espagne qui explique le silence et la disposition parfois négative des sources françaises envers eux. La présence saisonnière de navires de l'Espagne affecte l'historiographie de la région, dans le sens où elle doit être mise en relation avec l'établissement des structures coloniales françaises dans la baie des Chaleurs et sur la péninsule gaspésienne. Elle affecte aussi l'historiographie autochtone et métisse de la région, car les Basques de l'Espagne ont entretenu des contacts complexes avec les Amérindiens, et ce, de façon originale car ils n'étaient pas régis par le projet colonial tel qu'il caractérisait les rapports francoamérindiens. Enfin, le quasi silence des écrits au sujet des Basques dans la baie des Chaleurs au XVII ${ }^{\mathrm{e}}$ siècle accentue le rôle de l'archéologie et d'autres approches pour mieux comprendre la présence basque ancienne dans les zones identifiées par Detcheverry.

Le routier constitue une rare voix proprement basque dans l'histoire du golfe du Saint-Laurent au XVII ${ }^{e}$ siècle, et sa perspective inédite jette un nouvel éclairage sur les autres écrits portant sur les Basques et sur les pêcheries en général dans la baie des Chaleurs et sur le littoral gaspésien, au moment de l'ordonnance de la Marine de 1681 et de l'établissement des premières seigneuries gaspésiennes.

\section{ANNEXE: TRADUCTION DU DOCUMENT}

Dans le texte qui suit, nous avons mis le basque d'origine et notre traduction française en parallèle, afin de faciliter les comparaisons. Nous avons gardé l'orthographe d'origine de Detcheverry et notre traduction se veut la plus proche possible de l'original. Quand un mot basque précis nous apparaît significatif ou son sens incertain, nous l'avons ajouté entre crochets dans la traduction. Lorsque le sens précis d'une phrase ou d'une expression nous échappe, plutôt que de proposer une interprétation concise mais possiblement erronée, nous avons préféré conserver l'ambiguïté, en laissant aux chercheurs futurs le soin d'y apporter une lumière plus claire. Il en émerge, nous l'espérons, une idée du style de Detcheverry et de la complexité de ce premier texte professionnel en langue basque. 


\begin{tabular}{|l|}
\hline \multicolumn{1}{|c|}{ Detcheverry, 1677} \\
\hline $\begin{array}{l}\text { laquiteco duçu Forsemolueco punta eta caipesco } \\
\text { punta dire [p. 134...] nordest suduest haricen duçula } \\
\text { laurden bat estetic edo uestetic, eta dire hirur lecoa, } \\
\text { eta Caispeco puntac badu Irla chume bat puntan ez } \\
\text { hari sobera hurbil denbora calmequin, ceren erre- } \\
\text { mulia handia dabilla bira. }\end{array}$ \\
\hline $\begin{array}{l}\text { laquiteco duçu handic eta graberat edo unçiec } \\
\text { muillatcen duten leccurat bada lecoa chume bat eta } \\
\text { han amarratcen tuste unciac cayaren trebessean eta } \\
\text { finquatcen dute probiça leihorrean. }\end{array}$ \\
\hline
\end{tabular}

Halaber, baldin loan nahi baduçu fundamenerat edo dela amarra gabes edo berçe façoinnes, loanen çare istiborreco leihorra beçala apur bat amor eguiten dioçula haric eta ababorreco aldeco hareazco puntarainno, eta eguinnen dioçu amor haguits punta hari, ceren lehenic egorry behar baituçu chalupa buiaren ematerat ea non den ur chumearen punta, eta utcico duçu buya hura leihor aldetic, eta guero buya doblatu ondon joanen çare ababorreco alderat placer duçun beçain leihor, eta emanen duçu cable on bat edo ahalic hoberena noroesteco bayarat, eta berceac çure comoditaterat.

laquiteco duçu Irla de perceco suduesteco burua eta chegoanchisco punta daunzala nordest suduest harcen duçula laurden bat nortetic edo hegoatic, eta dire hamasey lecoa.

laquiteco duzu chegoanchisco puntary etçaiscola hurbildu behar hemeçorcy brassa baino gutiagotarat, ceren handic bi agustat eguin bainno lehen içaren duçu hamabi brassa Chegoanchisco costa gustian KaraKic iragan artean, eta hamaby brassetaric berce by agustaren burucots curry edo lau eta leccu batçubetan icusico duçu leihorrat çarela by lecoa, eta içanen duçu lau brassa eta hainnits lecutan menturas içaren çare lecoa baten arrastoa çabal, bachamarean chalupa enquailla daiteque guehien lecutan harea gogorra da chegoanchisco punta beraren gainnean harea pugubec urrun diraute eta içaren çare abisatuba chegoanchisco puntatic eta Karakik iragan artean leihorhec haguits dire arrassoac eta planac, ceinnetan ezpaita possible leihorric eçagutcea unguy, non estuçun chalupa leihorrat egorcen edo behartu hura eçagutuco dubenac peçabyaco leihorrac eçagutu, ceren norteco leihorrec mendy eçagutcecoac baitire, eta eçagutu ondoan leihor hetarico arruten arrespetura hobequienic eçagutcen dute; hartaracots
Traduction

Sache que la pointe de Forsemolue ${ }^{1}$ [pointe SaintPierre] et la pointe Caispe [cap Gaspé] gisent nordest $^{2}$ et sud-ouest quand tu prends un quart de l'est ou de l'ouest; et il y a 3 lieues [lekoa] $]^{3}$; et la pointe de Caispe a une très petite île; ne t'y approche pas trop par temps calme, parce qu'il y a un grand remous.

Sache que de là jusqu'à la grave ou à l'endroit où mouillent les bateaux [unziek], il y a 1 lieue juste; et les bateaux amarrent de travers [trabesean] au quai [kaia]; et ils fixent leur mouillage [probiza $\left.{ }^{4}\right]$ à terre.

Ainsi, si tu veux aller à Fundamen ${ }^{5}$ [Petite-Rivière], soit sans amarre ${ }^{6}$ ou d'une autre façon, tu iras comme [bezala] est la terre à tribord, tout en cédant un peu du côté de bâbord jusqu'à arriver à la pointe de sable, et tu feras attention de céder très fort à cette pointe-là [hari] ${ }^{7}$; car d'abord tu dois envoyer la chaloupe pour trouver la bouée [buia] et pour voir la pointe où il y a peu d'eau; et tu laisseras la bouée du côté de la terre; et après avoir doublé la bouée, tu iras vers le côté de bâbord à l'endroit à terre où tu voudras; et tu porteras un bon câble [cable], ou le meilleur possible, à la baie [baia] du nord-ouest, ainsi que tout ce dont tu as besoin pour ta commodité.

Sache que le cap [buru] sud-ouest de l'île de Percé [Bonaventure?] et la pointe de Chegoanchis [Miscou] gisent nord-est et sud-ouest, quand tu prends un quart du nord ou du sud, et il y a 16 lieues $^{8}$.

Sache qu'on ne doit pas approcher la pointe de Chegoanchis de moins de 18 brasses, parce que de là, avant de faire 2 agustas', tu auras 12 brasses tout au long de la côte de Chegoanchis jusqu'à avoir passé KaraKic [Caraquet]; et après avoir parcouru encore 2 ou 4 agustas, et par 12 brasses, tu verras que dans quelques endroits tu seras à 2 lieues de la terre et tu auras 4 brasses; il se peut que tu sois peut-être dans beaucoup d'endroits à 1 bonne lieue de la terre et par basse mer la chaloupe peut échouer [enkailla]; dans la plupart des endroits, le sable est dur [et] sur la pointe même de Chegoanchis, les bancs ${ }^{10}$ [pugubek] de sable vont loin; et tu seras averti que, de la pointe de Chegoanchis et jusqu'à avoir passé Karakik, beaucoup des terres sont rases et planes et il n'est pas possible de bien reconnaître [ezagutzea] la terre, si tu n'envoies pas la chaloupe à terre ou si tu n'engages pas quelqu'un qui reconnaît bien les terres de Peçabyac, car dans la terre au nord il y a des montagnes qu'on doit reconnaître. Et après les 
eman ditugu hemen hetaco arrutac, laquiteco duçu chequen ceina baita pecebiacoac grata eguiten duben leccuba, eta Chigoanchis daunza nort esu hartcen duçula aphur bat noroestetic, eta dire 12 leccoa. [p. $135 \ldots .$.

Halaber Pecebiac eta chegoanchis noroest suest eta dire $14 \mathrm{I}$. Halaber Pecebiac eta Karakic daunza nortesu eta dire sortcy edo bederatcy lecoa.

Halaber jaquin behar duçu Pecebiac eta estancia daunza edo Pecheguy daritçan baya nordest suduest, eta dire hamalau lecoa, bainan jaquiteco duçu menturas handic zerbait beharres joaten baçare estancya hura da, bayan barna, bainnan iaquiteco duçu noizere baitçare chaluparequin edo garrayoarequin tarte hercy batean eta baldin ioaten baçare bachamara denean, joanen çare sarcean istiborreco puntarat iendeen etchatcerat, ceren bachamarean chaluparic ezta iraganen baldin chanalen berrya espadaquy estanciarat eta estancia da punta hartaric bygarren senoan, eta ezta aguery haric eta bererat ioan artean istiborreco aldean eguiçu contu badela puntatic lecoa erdi bat.

laquiteco duçu baldin baçabilça luyetan haguits dabilçala goaya handiac, eta comunsquy esterat hartcen dubela nordestetic, eta baldin baçare segoanchisco aldetic es hurbil hemeçorcy braça baino gutiagotarat, eta baldin baçare norteco partetic ez hurbil hogoi eta borts brassa bainno gutiagotarat, baldin ioan behar baduçu Karakirat eta baldin gerthatcen baçare chegoanchisco aldetic eta calmatcen baçaitçu, hobequi eguinnen duçu joateas hemeçorcy brassetarat han muillatcen baduçu, ceren bercela goayac coratuco çaitu.

laquiteco duçu nahi baduçu muillatu edo sarthu chegoanchisen ioanen çare suduesteco punta suesterat equarry artean, eta muillatuco duçu hemeçorcy brassetan, eta handic hartuco duçu marea, eta denbora sartcecoa lehenetan ematen citusten buyac han sartcecots, bainan diote chanalaren entrada descubritus guerostic ioan behar dubela suduesteco punta bererat, eta bethy ur handia caussituco dubela, bainnan niry apredicençat leheneco çaharren leguea hobe çait buyen ematea: hango penagatic barnat sarthus guerostic ioan behar duçu muillatcerat avoir connues, on connaît mieux et avec beaucoup de respect les routes de cette terre. On donne ici les routes de ces lieux. Sache que Chequen [PortDaniel], qui est l'endroit où les gens de Pecebiac font le dégrat des poissons [grata egin] ${ }^{11}$, et Chigoanchis gisent nord et sud quand tu prends un peu du nordouest; il y a 12 lieues ${ }^{12}$.

Ainsi, Pecebiac et Chegoanchis gisent nord-ouest et sud-est; il y a 14 lieues $^{13}$. De la même manière, Pecebiac et Karakic gisent nord et sud et il y a 8 ou 9 lieues $^{14}$.

Ainsi, sache que Pecebiak et Estancia ${ }^{15}$, qui est dans la baie appelée Pecheguy [Népissiguit], gisent nordest sud-ouest et il y a 14 lieues $^{16}$; mais sache, si par hasard tu dois y aller, que cette Estancya-là [hura] est dans la baie; mais sache, si tu es avec une chaloupe ou un charroi ${ }^{17}$ [garraio], que tu arrives par un passage étroit; si tu y vas par basse mer, tu iras en entrant [sartzean] à la pointe au tribord jusqu'à arriver chez les gens, parce que par basse mer les chaloupes ne peuvent passer que si on sait qu'il y a un chenal pour arriver à Estancia; et ce lieu est dans la deuxième anse et on l'aperçoit seulement en $y$ arrivant; tu dois tenir compte que de tribord il y a de la pointe une demi-lieue.

Sache que, si tu marches par un vent contraire [luia], qu'il y a là de grands courants de marée ${ }^{18}$ [goaia] qui vont habituellement à l'est du nord-est; si tu es du côté [aldetik] de Segoanchis [Miscou], ne t'en approche pas de moins de 18 brasses; et si tu es de la part [partetik] du nord, ne t'en approche pas de moins de 25 brasses; si par hasard tu dois aller à Karakic et s'il t'arrive de venir du côté [aldetik] de Chegoanchis et le courant se calme, tu feras mieux d'aller à 18 brasses si tu y mouilles, sinon le courant de marée te fera échouer ${ }^{19}\left[\right.$ koratu ${ }^{20}$.

Sache que si tu veux mouiller ou entrer dans Chegouanchis, tu iras jusqu'à ce que tu aies la pointe du sud-ouest au sud-est, et tu mouilleras à 18 brasses et, de là, tu prendras la marée; et dans les anciens temps, ils mettaient des bouées pour y entrer, mais on dit que dès que l'on aperçoit l'entrée du canal, on doit aller à la même pointe du sud-ouest, où l'on trouvera toujours beaucoup d'eau; mais, quant à moi, je préfère la coutume des anciens pour les apprentis qui est de fournir des bouées, à cause des difficultés qui y existent; une fois entré, tu dois aller mouiller 
entradas den ababorreco punta arraso baten gericerat, edo lehenbicico punta meharraren guericerat, ceinna baita Pinnalac eguiten ditusten punta, eta quaya eguiten dutena, eta ioanen çare amarratcerat quaya eguiten den leccurat, eta hainnitcec ematen dute probiça leihorrat.

Halaber iaquin behar duçu nahi baduçu sarthu Karakiquen, lehenic egorrico duçu chalupa buyen chanalean ermaterat eta lehenbicico aingura muillatu behar duben leccuban ematerat (p. 136...), baiñnan chanallean hobe litesque by aldetaric ematea, çeren es baten beçala baita chanalla, eta chanallac badirau lecoa baten arrastoa arradarat ioan behar du hamaby brassetarat, eta handic hartu behar du haiçe largoa eta marea.

laquiteco duçu Karakico Irla haguits dela gaits eçagutçea, çeren ezpaita apart certhanetic, eta Irla bera oihana baitu çerthanac beçala.

Halaber nahi baduçu ioan Pecebiacat ioanen çare senady hartan edo portu hartan den leihorric haltoenaren trebesserat, çeiña baita sarcean ababorreco aldearequin, eta ethorrico çaitçu noroesta leihor handien haren gaiñnetic, eta muillatuco duçu leihorretic by agusta çabal, eta han çarela seguratçecots mendy handy haric eta grabaric artean bada barracho bat ceinnetan sartcen baitire chalupac. à l'abri d'une pointe rase qui est à bâbord à l'entrée, ou à l'abri de la première pointe fine, qui est la pointe où l'on fait les vigneaux [piñalak] et où l'on fait le quai [kaia], et tu iras amarrer au lieu où l'on fait le quai, et où plusieurs fixent un mouillage [probiza] à terre.

Sache que si tu veux entrer à Karakique, d'abord tu enverras la chaloupe pour trouver la bouée du chenal et trouver l'endroit où l'on doit jeter l'ancre; mais dans le chenal ce serait mieux d'aller d'un côté à l'autre puisque le chenal est comme un Z; et le chenal continue sur une lieue jusqu'à la rade [arrada]; et on doit aller à 12 brasses et, de là, on doit prendre le vent du large et la marée.

Sache que l'île Karakic est très difficile à reconnaître, parce qu'elle n'est pas à part de la terre ferme, et l'île même a un bois comme la terre ferme.

Ainsi, si tu veux aller à Pecebiac, tu iras dans cette anse [senadi] ou dans ce port-là de travers [trebeserat] de la terre plus haute qui est de côté de bâbord, et le nord-ouest t'en viendra par-dessus cette terre haute, et tu mouilleras à 2 bonnes agustas de la terre, et pour t'assurer que tu es là-bas, entre cette grande montagne et la grave il y a un barachois [barratxo bat] dans lequel entrent les chaloupes.

1. Forsemolue est dans la baie des Molues, entre la pointe Saint-Pierre et le rocher Percé.

2. En 2013, la déclinaison magnétique à Miscou est de 19 degrés (341 degrés).

3. En réalité 7,0 milles marins.

4. «Prois ou proiz; Cordage ou amarre amené à terre jusqu'à un arbre, un canon, une ancre ou un bollard lorsqu'on est mouillé à proximité. Italien; Provesa", Timoteo O’Scanlan, Diccionario Maritímo Español (Madrid, Museo Naval, 1974), 439.

5. Ce toponyme, tiré du latin, signifie «fondation» ou «base». Orotariko Euskal Hiztegia, op. cit., «fundamen». Le suffixe décliné basque -rat signifie que ce lieu est une destination.

6. L'amarre est un câble de mouillage amené à une ancre submergée ou à un quai. Ce terme générique est similaire au cable mentionné quelques lignes plus loin. Pierre Lhande, Dictionnaire BasqueFrançais (Paris, Gabriel Beauchesne, 1926), 38.

7. La langue basque contient trois gradations de distance pour les pronoms/adjectifs démonstratifs; hau (celui-ci), hori (celui-là) et hura (celui de là-bas, soit plus au loin). Ils sont déclinés au datif; honi/ horri/hari.

8. En réalité 31,2 milles marins.

9. Agusta; nous ne connaissons pas la longueur de cette unité de mesure. Elle peut être l'équivalent d'une «encablure» (environ 200 mètres), sinon d'un «trait d'archer» ou d'un «trait de bombarde» (environ 100 mètres); A. Sauer, Das “Seebuch"..., op. cit., 143-144; M. de Hoyarsabal, Voyages avantureux..., op. cit., 209.

10. Littéralement, «tourbillons» de sable.

11. Selon Nicolas Denys, les pêcheurs du "grand Paspec-biac» sèchent leur morue à une rivière près du cap du "petit Paspbec-biac», soit la rivière Bonaventure. Denys, Description géographique..., op. cit., 214-215. E. Jumeau, La grande baye de St. Laurens..., op. cit., indique la pointe Pepchidiachich (PetitPepchidia) à la pointe Sawyer, au sud-est de Bonaventure.

12. En réalité 24,3 milles marins. 
13. En réalité 28,1 milles marins.

14. En réalité 18,4 milles marins.

15. Estancia signifie domaine ou ranch; le sens est similaire à hacienda. En Argentine coloniale, les estancias des jésuites comportaient une population autochtone, et donc le sens s'apparente à reducción.

16. En réalité 27,8 milles marins.

17. Un charroi, dans la pêche terre-neuvienne du XVII ${ }^{e}$ siècle, était une embarcation plus grande qu'une chaloupe, servant au transport de morue. Il arborait deux voiles.

18. Il s'agit sans doute du courant de la marée descendante, c'est-à-dire du jusant.

19. Koratu peut provenir de l'espagnol escorar, c'est-à-dire accorer, gîter, échouer.

20. Detcheverry semble vouloir dire ici: «si tu arrives à Caraquet du côté de Miscou par étale [de pleine mer] et tu veux mouiller, il faut aller à 18 brasses, sinon le courant [du jusant] peut te faire échouer». 